\title{
Longitudinal wake of a bunch of suddenly accelerated electrons within the radiation formation zone
}

\author{
R. A. Bosch \\ Synchrotron Radiation Center, University of Wisconsin-Madison, 3731 Schneider Dr., Stoughton, Wisconsin 53589, USA
}

(Received 22 March 2007; published 2 May 2007)

\begin{abstract}
The longitudinal wake is considered for a bunch of electrons that are suddenly accelerated to an ultrarelativistic velocity. This wake describes the wake of forward transition radiation, and it approximates the edge-radiation wake of a bunch exiting a bending magnet. The wake is large within the radiation formation zone, where it provides resistive impedance. A comparison with the computed wake downstream of a bending magnet yields good agreement, indicating that our wake expressions may be used to approximate the wake without numerical computation. For schemes in which a bunch produced by laserplasma acceleration exits the plasma and then drives a free electron laser (FEL), the transition-radiation wake causes energy losses of many $\mathrm{MeV}$ that may affect the FEL process.
\end{abstract}

PACS numbers: 41.60.Ap, 52.38.Kd

\section{INTRODUCTION}

The longitudinal wake of an electron bunch may have a substantial effect upon the energy of the electrons. The variation of electron energies at the entrance of an undulator, and the change in electron energies when propagating through the undulator, may spoil the operation of a free electron laser (FEL). For an ultrarelativistic bunch emitted from plasma after laser-plasma acceleration, evaluation of this effect requires calculation of the transition-radiation wake within the radiation formation zone [1].

Starting with an expression for the longitudinal electric field of an electron that is suddenly deflected or decelerated [2], we obtain the wake of a bunch whose electrons are suddenly accelerated. This wake also describes forward transition radiation, and it approximates the wake of a bunch exiting a bending magnet at distances from the magnet edge exceeding the formation length of the magnet's ordinary synchrotron radiation.

Within the radiation formation zone, we calculate analytically the wake resulting from the bunch's coherent radiation. At sufficient distance from the location of the acceleration, the on-axis wake is resistive and inversely proportional to the distance from the location of the acceleration. Outside the formation zone, the coherent-radiation wake is much smaller.

A comparison with the wake downstream of a bending magnet yields good agreement. This suggests that our wake expressions may be useful for approximating the wake of a bunch compressor chicane. We consider the wake for schemes in which a bunch produced by laserplasma acceleration exits the plasma and then drives a vacuum ultraviolet (VUV) or x-ray FEL. In these cases, the wake causes energy losses of several MeV that may affect the FEL process.

\section{SUDDENLY STOPPED ELECTRON}

When an ultrarelativistic electron traveling in uniform motion along the $z$-axis is suddenly stopped at the origin, the radiation at wavelength $\lambda$, within the radiation formation zone $R \ll \lambda \gamma^{2} / 2 \pi$, is radially polarized with opening angle $\theta_{\text {open }} \approx(\lambda / R)^{1 / 2}$, where $R$ is the distance from the origin and $\gamma$ is the relativistic factor [2]. When an ultrarelativistic electron is stopped or deflected in a finite time, emitting radiation over a distance $d$ that is much smaller than the radiation formation length $\lambda \gamma^{2} / 2 \pi$, the radiation at wavelength $\lambda$ is well approximated by that of a suddenly stopped electron when $d \ll \lambda / \theta_{\text {open }}^{2} \approx R$, i.e., for $R \gg d$ [2].

An electron's electric field equals the sum of the Liénard-Wiechert acceleration and velocity fields. In SI units, the longitudinal acceleration field of an ultrarelativistic electron that travels along the $z$-axis until it is stopped at the origin at time $t=0$ is [2]

$$
\begin{aligned}
\tilde{E}_{a}(R, \theta, \omega) & =\int_{-\infty}^{\infty} E_{a}(R, \theta, t) e^{i \omega t} d t \\
& =e^{i k R} \frac{e}{4 \pi \varepsilon_{0} c R}\left[\frac{\beta \sin ^{2}(\theta)}{1-\beta \cos (\theta)}\right] \\
& \approx e^{i k R} \frac{e}{2 \pi \varepsilon_{0} c R}\left[\frac{(\gamma \theta)^{2}}{1+(\gamma \theta)^{2}}\right]
\end{aligned}
$$

Here, $\theta \ll \pi / 2$ is the observation angle with respect to the $z$-axis, $\omega$ is angular frequency, $e<0$ is the electron charge, $c$ is the speed of light, $\varepsilon_{0}$ is the permittivity of free space, $k=\omega / c$ is the wave number, and $\beta$ is the electron velocity divided by $c$. The longitudinal velocity field for $\theta \ll \pi / 2$ is [2] 


$$
\begin{aligned}
\tilde{E}_{v}(R, \theta, \omega)= & e^{i k R} \frac{e}{2 \pi \varepsilon_{0} c R} \frac{e^{i \pi R_{n}\left(1-\varphi^{2}\right)}}{\varphi} \\
& \times \int_{0}^{\varphi} \frac{1-x^{2}}{\left(1+x^{2}\right)^{2}} e^{i \pi R_{n} \varphi(x-1 / x)} d x,
\end{aligned}
$$

where $\varphi \equiv \gamma \theta$ and $R_{n} \equiv R / \lambda \gamma^{2}$. Outside the radiation formation zone $\left(R \gg \lambda \gamma^{2} / 2 \pi\right)$, the velocity field vanishes. Within the formation zone $\left(R \ll \lambda \gamma^{2} / 2 \pi\right)$, the velocity field is [2]

$$
\tilde{E}_{v}(R, \theta, \omega)=e^{i k R} \frac{e}{2 \pi \varepsilon_{0} c R}\left[\frac{1}{1+(\gamma \theta)^{2}}\right] .
$$

Summing the acceleration and velocity fields gives the longitudinal field within the formation zone [2]

$$
\tilde{E}(R, \theta, \omega)=e^{i k R} \frac{e}{2 \pi \varepsilon_{0} c R} .
$$

Outside the formation zone, the on-axis longitudinal field, given by Eq. (1), is zero. Consequently, the on-axis longitudinal field of a bunch of suddenly stopped electrons is largest within the formation zone, where it will accelerate any electrons that are present.

\section{SUDDENLY ACCELERATED ELECTRON}

The radiation field of an electron suddenly accelerated from rest is given by subtracting the field of a suddenly stopped electron from the Coulomb field of an electron in uniform motion [3]. Therefore, the radiation at wavelength $\lambda$ of an electron that is accelerated in a finite time, emitting radiation over a distance $d \ll \lambda \gamma^{2} / 2 \pi$, will be approximately described by the radiation from a sudden acceleration where $R \gg d$.

For an electron traveling on the $z$-axis, which passes through the origin at time $t=0$, the longitudinal Coulomb field expressed in coordinates $r=R \theta$ and $z$ is [4]

$$
\tilde{E}_{\mathrm{Coul}}(r, z, \omega)=e^{i k z} \exp \left(i k z / 2 \gamma^{2}\right)\left(\frac{-i e \omega}{2 \pi \varepsilon_{0} c^{2} \gamma^{2}}\right) K_{0}\left(\frac{|\omega| r}{c \gamma}\right) .
$$

Within the formation zone, subtracting Eq. (4) from Eq. (5) gives the longitudinal field of a suddenly accelerated electron

$$
\begin{aligned}
\tilde{E}(r, z, \omega)= & e^{i k z}\left[\left(\frac{-i e \omega}{2 \pi \varepsilon_{0} c^{2} \gamma^{2}}\right) K_{0}\left(\frac{|\omega| r}{c \gamma}\right)\right. \\
& \left.-\left(\frac{e}{2 \pi \varepsilon_{0} c}\right) \frac{\exp \left(i k \sqrt{z^{2}+r^{2}}-i k z\right)}{\sqrt{z^{2}+r^{2}}}\right] .
\end{aligned}
$$

\section{WAKE OF A BUNCH}

Consider a cylindrically symmetric bunch of $N$ electrons where each electron is suddenly accelerated from rest to an ultrarelativistic velocity at the $z=0$ plane. Let the bunch's current density through the $z=0$ plane at radius $r$ and time $t$ equal $N e \rho(r) f(t)$, where $2 \pi \int_{0}^{\infty} r d r \rho(r)=$ $\int_{-\infty}^{\infty} f(t) d t=1$. The form factor $F(\omega)$ equals $\int_{-\infty}^{\infty} f(t) \times$ $\exp (i \omega t) d t$. For a Gaussian bunch, $F(\omega)>0.6$ for $\lambda>$ $2 \pi \sigma_{z}$, where $\sigma_{z}=c \sigma_{t}$ is the bunch length, so that the bunch's coherent radiation is primarily composed of wavelengths exceeding $2 \pi \sigma_{z}$. The radiation formation zone for $\lambda=2 \pi \sigma_{z}$ therefore approximates the bunch's radiation formation zone [1], with formation length of $\left(2 \pi \sigma_{z}\right) \gamma^{2} / 2 \pi=\sigma_{z} \gamma^{2}$.

When the electrons in a bunch are accelerated in a finite time, emitting radiation over a distance $d \ll \sigma_{z} \gamma^{2}$, the bunch's radiation is primarily composed of wavelengths obeying $d \ll \lambda \gamma^{2} / 2 \pi$, and therefore well approximated by the radiation of suddenly accelerated electrons where $R \gg d$.

In the frequency domain, the longitudinal electric field of a bunch of suddenly accelerated electrons is

$$
\tilde{E}_{N}(r, z, \omega)=N F(\omega) \int_{0}^{2 \pi} d \phi^{\prime} \int_{0}^{\infty} r^{\prime} d r^{\prime} \rho\left(r^{\prime}\right) \tilde{E}(\Delta r, z, \omega),
$$

where $(\Delta r)^{2}=\left(r-r^{\prime} \cos \phi^{\prime}\right)^{2}+\left(r^{\prime} \sin \phi^{\prime}\right)^{2}$. The longitudinal wake experienced by an electron traveling in the $z$-direction, that passes through the $z=0$ plane at time $\Delta t$, is

$$
\begin{aligned}
W(r, z, \Delta t)= & E_{N}(r, z, t=z / \beta c+\Delta t) \\
= & \frac{1}{2 \pi} \int_{-\infty}^{\infty} \exp [-i \omega(z / \beta c+\Delta t)] \\
& \times \tilde{E}_{N}(r, z, \omega) d \omega .
\end{aligned}
$$

For an ultrarelativistic electron in the formation zone, the on-axis wake of the bunch is therefore

$$
\begin{aligned}
W(r= & 0, z, \Delta t) \\
= & N \int_{-\infty}^{\infty} F(\omega) e^{-i \omega \Delta t} d \omega \int_{0}^{\infty} r^{\prime} d r^{\prime} \rho\left(r^{\prime}\right) \\
& \times\left[\left(\frac{-i e \omega}{2 \pi \varepsilon_{0} c^{2} \gamma^{2}}\right) K_{0}\left(\frac{|\omega| r^{\prime}}{c \gamma}\right)-\left(\frac{e}{2 \pi \varepsilon_{0} c}\right)\right. \\
& \left.\times \frac{\exp \left(i k \sqrt{z^{2}+r^{\prime 2}}-i k z\right)}{\sqrt{z^{2}+r^{\prime 2}}}\right] .
\end{aligned}
$$

The wake is the sum of a Coulomb (space charge) wake and a coherent-radiation wake, where the Coulomb wake is

$$
\begin{aligned}
W_{\text {Coul }}(0, z, \Delta t)= & N \int_{-\infty}^{\infty} F(\omega) e^{-i \omega \Delta t} d \omega \int_{0}^{\infty} r^{\prime} d r^{\prime} \rho\left(r^{\prime}\right) \\
& \times\left[\left(\frac{-i e \omega}{2 \pi \varepsilon_{0} c^{2} \gamma^{2}}\right) K_{0}\left(\frac{|\omega| r^{\prime}}{c \gamma}\right)\right]
\end{aligned}
$$

and the coherent radiation wake is 


$$
\begin{aligned}
W_{\mathrm{CR}}(0, z, \Delta t)= & N \int_{-\infty}^{\infty} F(\omega) e^{-i \omega \Delta t} d \omega \int_{0}^{\infty} r^{\prime} d r^{\prime} \rho\left(r^{\prime}\right) \\
& \times\left[\left(\frac{-e}{2 \pi \varepsilon_{0} c}\right) \frac{\exp \left(i k \sqrt{z^{2}+r^{\prime 2}}-i k z\right)}{\sqrt{z^{2}+r^{\prime 2}}}\right] \\
= & \left(\frac{-N e}{\varepsilon_{0} c}\right) \int_{0}^{\infty} \frac{r^{\prime} d r^{\prime} \rho\left(r^{\prime}\right)}{\sqrt{z^{2}+r^{\prime 2}}} \\
& \times f\left(\Delta t-\frac{\sqrt{z^{2}+r^{\prime 2}}-z}{c}\right)
\end{aligned}
$$

The coherent-radiation wake is positive, so that it decelerates electrons.

For a uniform radial density distribution within a bunch radius of $r_{b}\left[\rho(r)=1 / \pi r_{b}^{2}\right.$ for $\left.r<r_{b}\right]$, the wakes are

$$
\begin{aligned}
W_{\text {Coul }}(0, z, \Delta t)= & \frac{-N e}{2 \pi^{2} \varepsilon_{0} r_{b}^{2}} \int_{-\infty}^{\infty} F(\omega) e^{-i \omega \Delta t}\left(\frac{i}{\omega}\right) \\
& \times\left[1-\frac{|\omega| r_{b}}{c \gamma} K_{1}\left(\frac{|\omega| r_{b}}{c \gamma}\right)\right] d \omega
\end{aligned}
$$

and

$$
W_{\mathrm{CR}}(0, z, \Delta t)=\frac{-N e}{2 \pi^{2} \varepsilon_{0} r_{b}^{2}} \int_{-\infty}^{\infty} F(\omega) e^{-i \omega \Delta t} \frac{\exp \left(i k \sqrt{z^{2}+r_{b}^{2}}-i k z\right)-1}{i c k} d \omega
$$

For $k r_{b} \leq 1$, the integrand of Eq. (12) [5] is much smaller than that of Eq. (13), where $z \ll \lambda \gamma^{2} /\left[2 \pi \ln \left(\lambda \gamma / 2 \pi r_{b}\right)\right]$. Thus, when $r_{b} \leq \sigma_{z}$, the Coulomb wake is much smaller than the coherent-radiation wake where

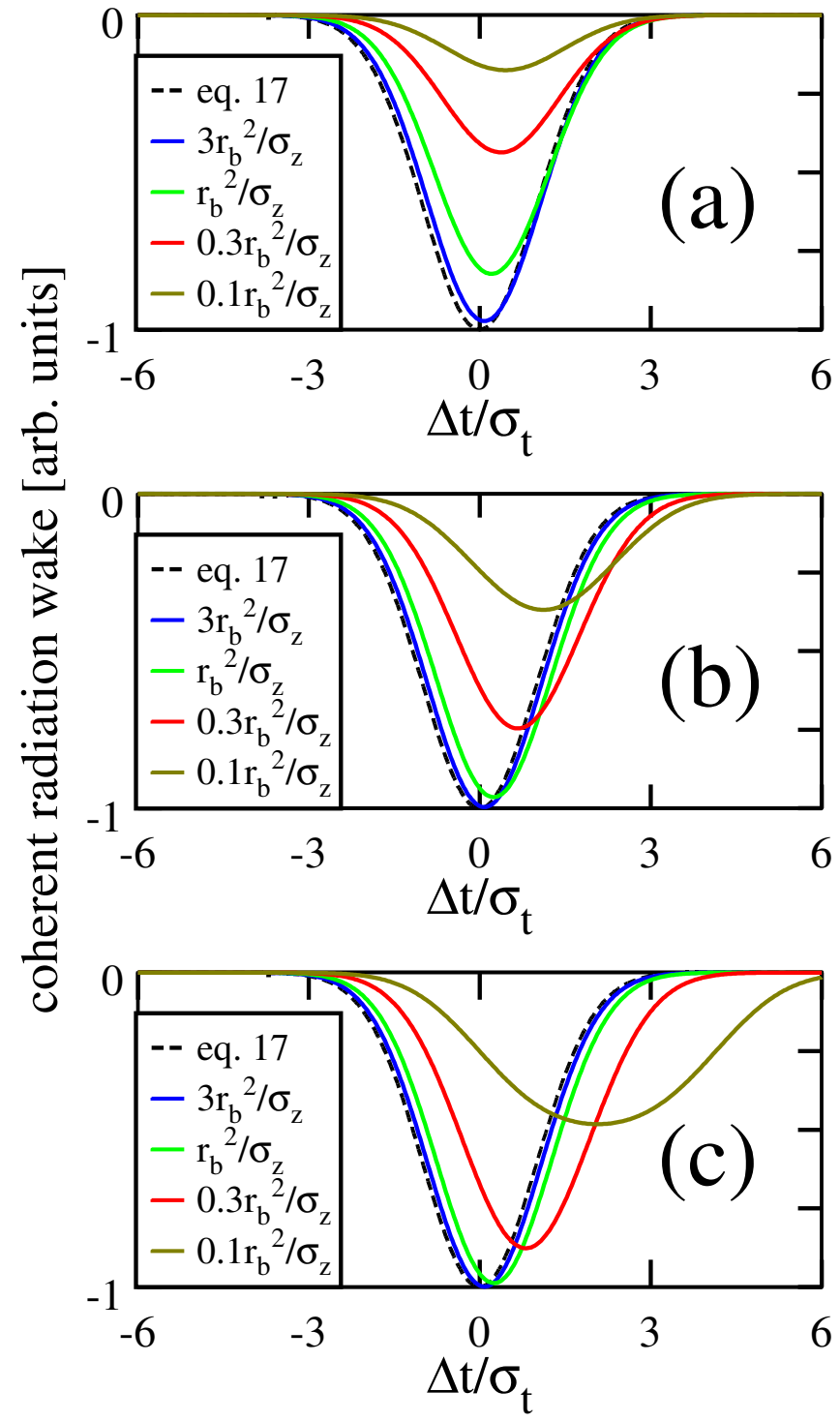

FIG. 1. (Color) For bunches with a Gaussian temporal distribution, the coherent-radiation wake of suddenly accelerated electrons is computed numerically from Eq. (15) and approximated by Eq. (17). The wakes are computed for four values of the axial coordinate $z$, and normalized to the height of the approximate wake given by Eq. (17). Here, a negative wake corresponds to energy loss; a positive $\Delta t$ describes the tail of the bunch. (a) $r_{b}=$ $\sigma_{z}$. (b) $r_{b}=3 \sigma_{z}$. (c) $r_{b}=10 \sigma_{z}$. 


$$
z \ll \sigma_{z} \gamma^{2} / \ln \left(\sigma_{z} \gamma / r_{b}\right) .
$$

When $\sigma_{z}<r_{b} \ll \sigma_{z} \gamma$, the Coulomb wake is much smaller than the coherent-radiation wake where $r_{b}^{2} / \sigma_{z}<$ $z \ll \sigma_{z} \gamma^{2} / \ln \left(\sigma_{z} \gamma / r_{b}\right)$.

Equation (13) may be written as

$$
W_{\mathrm{CR}}(0, z, \Delta t)=\frac{-N e}{\pi \varepsilon_{0} r_{b}^{2}} \int_{\Delta t-\left[\left(\sqrt{z^{2}+r_{b}^{2}}-z\right) / c\right]}^{\Delta t} f(t) d t .
$$

For a Gaussian temporal distribution $f(t), W_{\mathrm{CR}}(0, z, \Delta t)=$ $\left(-N e / 2 \pi \varepsilon_{0} r_{b}^{2}\right)\left\{\operatorname{erf}\left[\Delta t /\left(\sqrt{2} \sigma_{t}\right)\right]-\operatorname{erf}\left[\left(\Delta t-\sqrt{z^{2}+r_{b}^{2}} / c+\right.\right.\right.$ $\left.\left.z / c) /\left(\sqrt{2} \sigma_{t}\right)\right]\right\}$.

For $r_{b} \ll \sigma_{z}, f(t)$ is nearly constant over the integration region of Eq. (15), so that the coherent-radiation wake may be written in terms of the beam current profile $I(\Delta t)=$ $N e f(\Delta t)<0$ as

$$
\begin{aligned}
W_{\mathrm{CR}}(0, z, \Delta t) & =\left(\frac{-N e}{\pi \varepsilon_{0} r_{b}^{2}}\right)\left[\frac{\sqrt{z^{2}+r_{b}^{2}}-z}{c}\right] f(\Delta t) \\
& =\frac{-I(\Delta t)}{\pi \varepsilon_{0} c r_{b}^{2}}\left[\sqrt{z^{2}+r_{b}^{2}}-z\right] .
\end{aligned}
$$

Since the wake is proportional to the beam current, it is resistive with resistance per unit length of $\left(\sqrt{z^{2}+r_{b}^{2}}-\right.$ $z) /\left(\pi \varepsilon_{0} c r_{b}^{2}\right)$. For $z \gg r_{b}$, the wake is inversely proportional to the distance from the location of the acceleration

$$
W_{\mathrm{CR}}(0, z, \Delta t) \approx \frac{-I(\Delta t)}{2 \pi \varepsilon_{0} c z},
$$

with resistance per unit length of $\left(2 \pi \varepsilon_{0} c z\right)^{-1}=Z_{0} /(2 \pi z)$, where $Z_{0}=\left(\varepsilon_{0} c\right)^{-1}=120 \pi \Omega$ is the impedance of free space.

For $r_{b} \geq \sigma_{z}$, Eq. (17) applies where $z \gg r_{b}^{2} / \sigma_{z}$. Where $z \ll r_{b}^{2} / \sigma_{z}$, the wake is smaller than that given by Eq. (17), and its peak is shifted towards the tail of the bunch. For a bunch with a Gaussian temporal distribution and $r_{b} \geq \sigma_{z}$, wakes computed from Eq. (15) are plotted in Fig. 1, confirming that Eq. (17) applies if and only if $z \gg r_{b}^{2} / \sigma_{z}$. To conform to convention, the opposite of each computed wake is plotted so that a negative wake corresponds to energy loss.

The above wakes describe a bunch in which each electron is accelerated at the $z=0$ plane. For a bunch in which each electron is suddenly accelerated at the same time $t=$ 0 , Eq. (13) applies for $z \gg \max \left(\sigma_{z}, r_{b}\right)$, while Eq. (17) applies for $z \gg \max \left(\sigma_{z}, r_{b}^{2} / \sigma_{z}\right)$.

\section{SHIELDING}

Our equations for the formation-zone wake apply in free space. Consider a bunch traveling parallel to a conducting plate located at a distance $h / 2$ from the axis, where $h / 2 \gg$ $r_{b}$. We represent the conducting plate by an oppositely charged image bunch traveling at distance $h$ from the axis. For $z \gg h$, the on-axis coherent-radiation wake of the image bunch is given by Eqs. (7) and (8):

$$
\begin{aligned}
W_{\mathrm{CR}}(0, z, \Delta t) \approx & \frac{N}{2 \pi} \int_{-\infty}^{\infty} F(\omega) e^{-i \omega \Delta t} d \omega\left[\left(\frac{e}{2 \pi \varepsilon_{0} c}\right)\right. \\
& \left.\times \frac{\exp \left(i k \sqrt{z^{2}+h^{2}}-i k z\right)}{\sqrt{z^{2}+h^{2}}}\right] .
\end{aligned}
$$

For $z \gg h^{2} / 2 \pi \sigma_{z}$, the exponential term in Eq. (18) nearly equals one when the form factor $F(\omega)$ is $\sim 1$. Consequently, the image bunch's wake is opposite to the bunch's wake, given by Eq. (17). For $z \gg$ $\max \left(h, h^{2} / 2 \pi \sigma_{z}\right)$, the bunch's longitudinal wake is canceled, or "shielded," by a conducting plate, such as the wall of a vacuum chamber of height $h$. This is the same region where the formation-zone transverse coherentradiation field is shielded [6].

Our expressions for the longitudinal coherent-radiation wake [Eqs. (11) and (13)-(17)] apply for $z \ll$ $\min \left[\sigma_{z} \gamma^{2}, \max \left(h, h^{2} / 2 \pi \sigma_{z}\right)\right]$. For larger values of $z$, the on-axis coherent-radiation wake is relatively small.

\section{INTEGRATED WAKE}

When calculating the integrated wake of a bunch that is accelerated in a finite time, emitting radiation over a distance $d \ll \sigma_{z} \gamma^{2}$, we restrict our integration to values of $z$ where the wake is well approximated by the formationzone wake of suddenly accelerated electrons: $d<z \ll$ $\min \left[\sigma_{z} \gamma^{2}, \max \left(h, h^{2} / 2 \pi \sigma_{z}\right)\right]$. Our integrated wake expressions do not include the wake in the region where the bunch is accelerated, and they do not apply outside the formation zone. The integrated wake of coherent radiation as a bunch travels from axial coordinate $d$ to axial coordinate $z$ is, from Eq. (15),

$$
\begin{aligned}
\Delta V_{\mathrm{CR}}(r=0, z, \Delta t) \equiv & \int_{d}^{z} W_{\mathrm{CR}}\left(0, z^{\prime}, \Delta t\right) d z^{\prime} \\
= & \left(\frac{-N e}{\pi \varepsilon_{0} r_{b}^{2}}\right) \int_{d}^{z} d z^{\prime} \\
& \times \int_{\Delta t-\left[\left(\sqrt{z^{12}+r_{b}^{2}}-z^{\prime}\right) / c\right]}^{\Delta t} f(t) d t .
\end{aligned}
$$

For a Gaussian temporal distribution $f(t)$, Eq. (19) becomes $\quad \Delta V_{\mathrm{CR}}(0, z, \Delta t)=\left(-N e / 2 \pi \varepsilon_{0} r_{b}^{2}\right) \times$ $\int_{d}^{z} d z^{\prime}\left\{\operatorname{erf}\left[\Delta t /\left(\sqrt{2} \sigma_{t}\right)\right]-\operatorname{erf}\left[\left(\Delta t-\sqrt{z^{\prime 2}+r_{b}^{2}} / c+z^{\prime} / c\right) /\right.\right.$ $\left.\left.\left(\sqrt{2} \sigma_{t}\right)\right]\right\}$.

For a bunch with $r_{b} \ll \sigma_{z}$, the on-axis integrated wake from Eqs. (16) and (19) is [7] 


$$
\Delta V_{\mathrm{CR}}(r=0, z, \Delta t)=\int_{d}^{z} \frac{-I(\Delta t)}{\pi \varepsilon_{0} c r_{b}^{2}}\left[\sqrt{z^{\prime 2}+r_{b}^{2}}-z^{\prime}\right] d z^{\prime}=\frac{-I(\Delta t)}{2 \pi \varepsilon_{0} c}\left[\begin{array}{l}
\frac{z}{r_{b}} \sqrt{1+\left(\frac{z}{r_{b}}\right)^{2}}+\ln \left[\frac{z}{r_{b}}+\sqrt{1+\left(\frac{z}{r_{b}}\right)^{2}}\right]-\left(\frac{z}{r_{b}}\right)^{2} \\
-\frac{d}{r_{b}} \sqrt{1+\left(\frac{d}{r_{b}}\right)^{2}}-\ln \left[\frac{d}{r_{b}}+\sqrt{1+\left(\frac{d}{r_{b}}\right)^{2}}\right]+\left(\frac{d}{r_{b}}\right)^{2}
\end{array}\right]
$$

For $d \gg r_{b}$, the integrated wake is independent of the bunch radius $r_{b}$ and depends logarithmically upon the value of $d$, equaling $\left[-I(\Delta t) / 2 \pi \varepsilon_{0} c\right] \ln (z / d)$. For $d \ll$ $r_{b}<z$, the integrated wake is independent of $d$ and nearly equal to $\left[-I(\Delta t) /\left(2 \pi \varepsilon_{0} c\right)\right]\left[\ln \left(z / r_{b}\right)+1.193\right]$.

For a bunch with $r_{b} \geq \sigma_{z}$, the wake is resistive and approximated by Eq. (17) for $z>\max \left(d, r_{b}^{2} / \sigma_{z}\right)$, giving

$$
\begin{aligned}
\Delta V_{\mathrm{CR}}(0, z, \Delta t) & \approx \int_{\max \left(d, r_{b}^{2} / \sigma_{z}\right)}^{z} \frac{-I(\Delta t)}{2 \pi \varepsilon_{0} c z^{\prime}} d z^{\prime} \\
& =\frac{-I(\Delta t)}{2 \pi \varepsilon_{0} c} \ln \left[\frac{z}{\max \left(d, r_{b}^{2} / \sigma_{z}\right)}\right] .
\end{aligned}
$$

For $d \gg r_{b}^{2} / \sigma_{z}$, the integrated wake is independent of the bunch radius $r_{b}$ and depends logarithmically upon the value of $d$, equaling $\left[-I(\Delta t) / 2 \pi \varepsilon_{0} c\right] \ln (z / d)$. For $d \ll$ $r_{b}^{2} / \sigma_{z}$, the integrated wake is independent of $d$. In this case, Eq. (21) does not include the contributions of the smaller wake where $z^{\prime} \leq r_{b}^{2} / \sigma_{z}$. For a Gaussian temporal distribution $f(t)$, numerical evaluation of Eq. (19), which includes these small wake contributions, gives an integrated wake nearly equal to $\left[-I(\Delta t) /\left(2 \pi \varepsilon_{0} c\right)\right] \times$ $\left[\ln \left(z \sigma_{z} / r_{b}^{2}\right)+1.193\right]$ for $z \gg r_{b}^{2} / \sigma_{z}$ and $r_{b} \approx \sigma_{z}$. When $r_{b} \gg \sigma_{z}$, wake contributions from $z^{\prime} \leq r_{b}^{2} / \sigma_{z}$ cause the peak of the integrated wake to be noticeably shifted towards the tail of the bunch.

Thus, for any value of $r_{b} / \sigma_{z}$, the integrated wake has little dependence upon $d$ when

$$
d \ll \max \left(r_{b}, r_{b}^{2} / \sigma_{z}\right) .
$$

In this case, the integrated wake is well described by integrating the wake of suddenly accelerated electrons from axial coordinate 0 to axial coordinate $z$. For $r_{b} \leq$ $\sigma_{z}$, the integrated coherent-radiation wake for $z>$ $\max \left(r_{b}, r_{b}^{2} / \sigma_{z}\right)$ nearly equals

$$
\Delta V_{\mathrm{CR}}(0, z, \Delta t)=\frac{-I(\Delta t)}{2 \pi \varepsilon_{0} c}\left\{\ln \left[\frac{z}{\max \left(r_{b}, r_{b}^{2} / \sigma_{z}\right)}\right]+1.193\right\} \text {. }
$$

Equation (23) describes a resistive integrated wake, with resistance of $\left(Z_{0} / 2 \pi\right)\left\{\ln \left[z / \max \left(r_{b}, r_{b}^{2} / \sigma_{z}\right)\right]+1.193\right\}$. In Fig. 2, integrated wakes are computed from Eq. (19), confirming that Eq. (23) applies for $r_{b} \leq \sigma_{z}$. When $r_{b}>\sigma_{z}$, the peak of the integrated wake is shifted towards the tail of the bunch and the integrated wake develops a long tail that is not described by Eq. (23); the central portion of the integrated wake is approximated by Eq. (23) for $z \gg$ $\max \left(r_{b}, r_{b}^{2} / \sigma_{z}\right)$.
Within the formation zone, Eq. (23) indicates that the integrated wake for $d=0$ depends logarithmically upon $r_{b}$ as $r_{b} \rightarrow 0$, as it does outside the formation zone [1]. For a bunch with a pancake shape in the beam frame $\left(r_{b}>\sigma_{z} \gamma\right)$, $r_{b}^{2} / \sigma_{z}$ exceeds the formation length $\sigma_{z} \gamma^{2}$, and the inte-
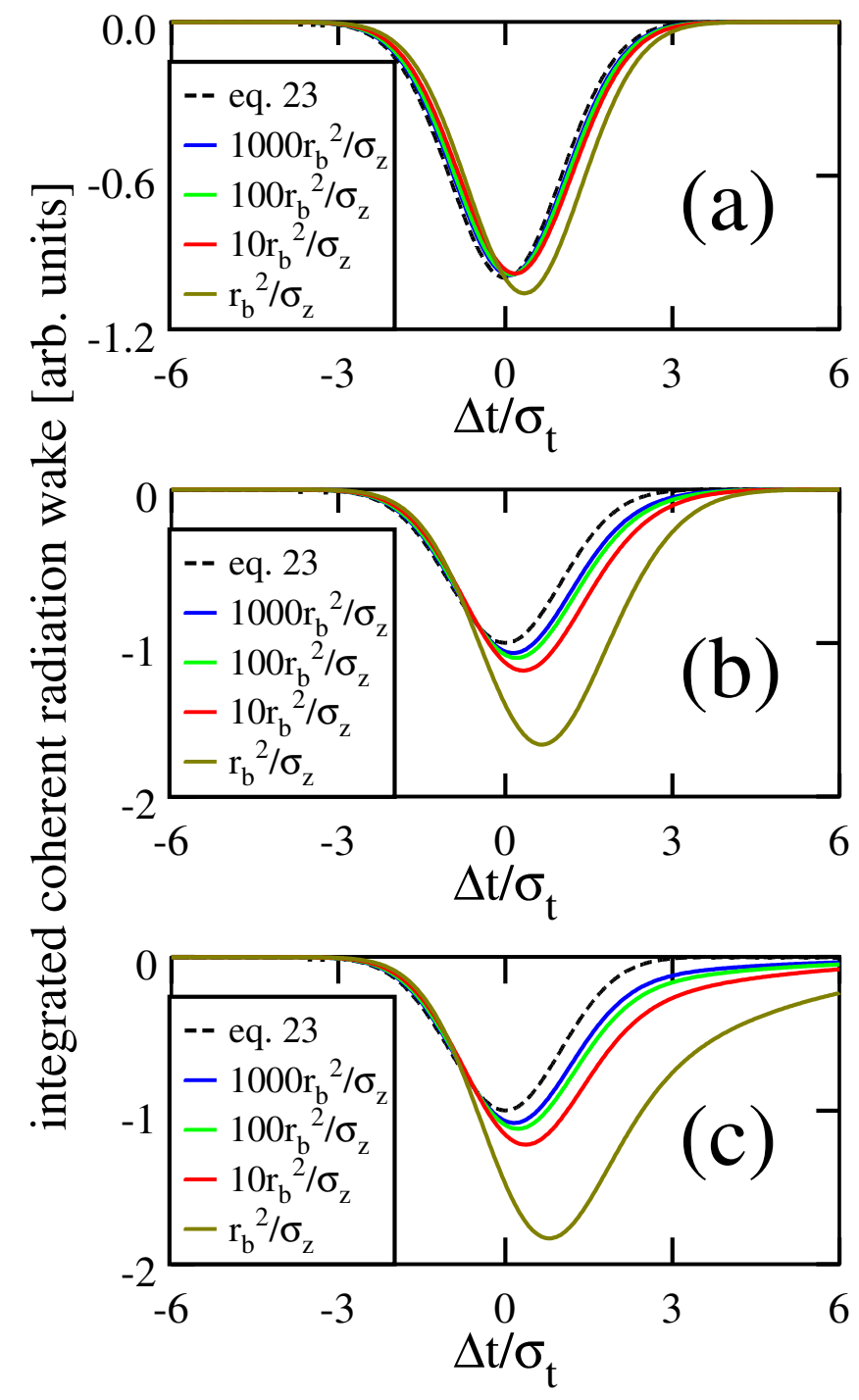

FIG. 2. (Color) For bunches with a Gaussian temporal distribution, the integrated coherent-radiation wake of suddenly accelerated electrons is computed numerically from Eq. (19) and approximated by Eq. (23). The integrated wakes are computed for four values of the axial coordinate $z$, and normalized to the height of the approximate integrated wake given by Eq. (23). Here, a negative wake corresponds to energy loss; a positive $\Delta t$ describes the tail of the bunch. (a) $r_{b}=\sigma_{z}$. (b) $r_{b}=3 \sigma_{z}$. (c) $r_{b}=10 \sigma_{z}$. 
grated wake within the formation zone is nearly zero (with impedance $\left.\ll Z_{0}\right)$. This is also the case outside the formation zone [1].

Our integrated wake expressions apply for $d<z \ll$ $\min \left[\sigma_{z} \gamma^{2}, \max \left(h, h^{2} / 2 \pi \sigma_{z}\right)\right]$. To estimate the integrated wake for larger values of $z$, one may set $z=$ $\min \left[\sigma_{z} \gamma^{2}, \max \left(h, h^{2} / 2 \pi \sigma_{z}\right)\right]$ in Eqs. (19)-(21) and (23), with approximation error of $\sim I(\Delta t) /\left[2 \pi \varepsilon_{0} c\right]=$ $I(\Delta t) Z_{0} /[2 \pi]$, which gives approximation error in the integrated impedance of $\sim 60 \Omega$.

\section{EDGE RADIATION}

Consider a bunch exiting a hard-edged bending magnet in which the orbit's radius of curvature is $\rho$, for the case where the formation length of ordinary synchrotron radiation $\rho^{2 / 3}\left(2 \pi \sigma_{z}\right)^{1 / 3}$ is small compared to $\sigma_{z} \gamma^{2}$. When the length of the magnet exceeds the formation length of ordinary synchrotron radiation, the transient edgeradiation wake from the magnet entrance does not affect the bunch after it exits the magnet. An electron's radiation at wavelength $\lambda$ is approximated by the formation-zone radiation from a sudden acceleration at distances $R$ from the magnet exit obeying $d \ll R \ll \lambda \gamma^{2} / 2 \pi$, where $d=$ $\rho(\lambda / R)^{1 / 2}$ is the distance required to deflect an electron through the angle $(\lambda / R)^{1 / 2}$ [2]. This gives $\rho^{2 / 3} \lambda^{1 / 3} \ll$ $R \ll \lambda \gamma^{2} / 2 \pi$. Thus, the coherent radiation of a bunch is approximated by the radiation of suddenly accelerated electrons, and referred to as "edge radiation," where $R$ exceeds the formation length of ordinary synchrotron radiation $\rho^{2 / 3}\left(2 \pi \sigma_{z}\right)^{1 / 3}$.

Let the axial coordinate $z$ equal zero at the magnet exit. Then, for $z \gg d=\rho^{2 / 3}\left(2 \pi \sigma_{z}\right)^{1 / 3}$, the coherent-radiation wake equals the wake from suddenly accelerated electrons. When $d=\rho^{2 / 3}\left(2 \pi \sigma_{z}\right)^{1 / 3} \gg \max \left(r_{b}, r_{b}^{2} / \sigma_{z}\right)$, the integrated on-axis wake for $z \ll$ $\min \left[\sigma_{z} \gamma^{2}, \max \left(h, h^{2} / 2 \pi \sigma_{z}\right)\right]$ is approximately

$$
\begin{aligned}
\Delta V_{\mathrm{CR}}(0, z, \Delta t) & \approx \int_{\rho^{2 / 3}\left(2 \pi \sigma_{z}\right)^{1 / 3}}^{z} \frac{-I(\Delta t)}{2 \pi \varepsilon_{0} c z^{\prime}} d z^{\prime} \\
& =\frac{-I(\Delta t)}{2 \pi \varepsilon_{0} c} \ln \left[\frac{z}{\rho^{2 / 3}\left(2 \pi \sigma_{z}\right)^{1 / 3}}\right] .
\end{aligned}
$$

Equation (24) is the integrated wake of formation-zone edge radiation, which does not include contributions from the wake within the bending magnet or the wake within a distance $\rho^{2 / 3}\left(2 \pi \sigma_{z}\right)^{1 / 3}$ of the magnet edge. Equation (24) describes a resistive integrated wake, with resistance of $\left(Z_{0} / 2 \pi\right) \ln \left\{z /\left[\rho^{2 / 3}\left(2 \pi \sigma_{z}\right)^{1 / 3}\right]\right\}$.

To estimate the integrated wake when $z$ exceeds the formation length $\sigma_{z} \gamma^{2}$, one may let $z=\sigma_{z} \gamma^{2}$ in Eq. (24), obtaining an integrated edge-radiation wake of $\left[-I(\Delta t) /\left(3 \pi \varepsilon_{0} c\right)\right]\left[\ln \left(\sigma_{z} \gamma^{3} / \rho\right)-\ln (\sqrt{2 \pi})\right]$, which gives estimated edge-radiation impedance of $\left[Z_{0} /(3 \pi)\right] \times$ $\left[\ln \left(\sigma_{z} \gamma^{3} / \rho\right)-\ln (\sqrt{2 \pi})\right]$. For a rectangular bunch with peak current of $I_{0}$ and spatial bunch length of $l_{\mathrm{b}}$, the bunch's energy loss from this estimated impedance is $\left(l_{\mathrm{b}} / c\right) I_{0}^{2}\left[Z_{0} /(3 \pi)\right]\left[\ln \left(l_{\mathrm{b}} \gamma^{3} / \rho\right)-\ln (2 \sqrt{6 \pi})\right]$, in approximate agreement with the edge-radiation energy loss of $\left(l_{\mathrm{b}} / c\right) I_{0}^{2}\left[Z_{0} /(3 \pi)\right]\left[\ln \left(l_{b} \gamma^{3} / \rho\right)-4\right]$ obtained in Ref. [8].

Within the formation zone, we compared the wake of suddenly accelerated electrons with computed radiation wakes for a $1 \mathrm{nC}, 150 \mathrm{MeV}$ Gaussian bunch with $\sigma_{z}=$ $50 \mu \mathrm{m}$ undergoing a $19^{\circ}$ bend or $3.8^{\circ}$ bend with radius of curvature $\rho=1.5 \mathrm{~m}[9,10]$. In this example, $\sigma_{z} \gamma^{2}=$ $4.3 \mathrm{~m}$. For $z>\rho^{2 / 3}\left(2 \pi \sigma_{z}\right)^{1 / 3}=89 \mathrm{~mm}$, the computed radiation wakes are well described by Eq. (17), while the integrated wake is in approximate agreement with Eq. (24).

For a $19^{\circ}$ bending magnet, whose length is $5.6 \rho^{2 / 3}\left(2 \pi \sigma_{z}\right)^{1 / 3}$, the integrated edge-radiation wake is comparable to the integrated coherent-radiation wake within the bending magnet [9]. For a small bending angle of $3.8^{\circ}$, where the magnet length equals $1.1 \rho^{2 / 3}\left(2 \pi \sigma_{z}\right)^{1 / 3}$, the integrated wake of edge radiation exceeds the integrated coherent-radiation wake within the bending magnet [9], so that Eq. (24) approximates the total integrated coherent-radiation wake.

\section{FORWARD TRANSITION RADIATION FROM LASER-PLASMA ACCELERATION}

In paraxial approximation, the forward transition radiation from a perfect conductor is equivalent to the radiation from suddenly accelerated electrons. For a bunch exiting plasma after laser-plasma acceleration, the emission of transition radiation primarily occurs within the distance $d \sim 2.5 \mu \mathrm{m}$ over which the plasma's index of refraction changes from 0.8 to 1.0 [11]. We expect this wake to be described by the wake of suddenly accelerated electrons for $R \gg d$, i.e., for $z \gg 2.5 \mu \mathrm{m}$.

Consider a test-case tabletop VUV FEL scenario [12] in which a bunch is emitted from plasma with $r_{b} \approx \sigma_{z}=$ $1 \mu \mathrm{m}$, peak current of $50 \mathrm{kA}$, and energy of $150 \mathrm{MeV}$. After propagating to $z=4 \mathrm{~cm}$, the bunch is focused over $10 \mathrm{~cm}$ by a quadrupole triplet to form a collimated beam with $r_{b} \approx 30 \mu \mathrm{m}$ and $\sigma_{z} \approx 1 \mu \mathrm{m}$. At $z=15 \mathrm{~cm}$, the bunch enters an undulator with length of about $0.8 \mathrm{~m}$. We expect the coherent transition-radiation wake to be determined by the bunch dimensions as it exits the plasma, with radiation formation length of $\sigma_{z} \gamma^{2}=8.6 \mathrm{~cm}$. For a Gaussian temporal distribution, letting $d=2.5 \mu \mathrm{m}, r_{b}=$ $1 \mu \mathrm{m}$, and $z=3 \mathrm{~cm}$ in Eq. (19) gives the integrated coherent-radiation wake $1 \mathrm{~cm}$ upstream of the quadrupole triplet. We computed the integrated Coulomb (longitudinal space charge) wake by neglecting the transverse expansion of the bunch, i.e., by letting $r_{b}=1 \mu \mathrm{m}$ in Eq. (12). The results, shown in Fig. 3(a), indicate that the coherentradiation wake dominates, resulting in a peak decelerating wake of $25 \mathrm{MV}$. To decrease the coherent-radiation wake by shielding within the formation zone would require a vacuum-chamber height less than $\sigma_{z} \gamma \sqrt{2 \pi}=0.7 \mathrm{~mm}$. 


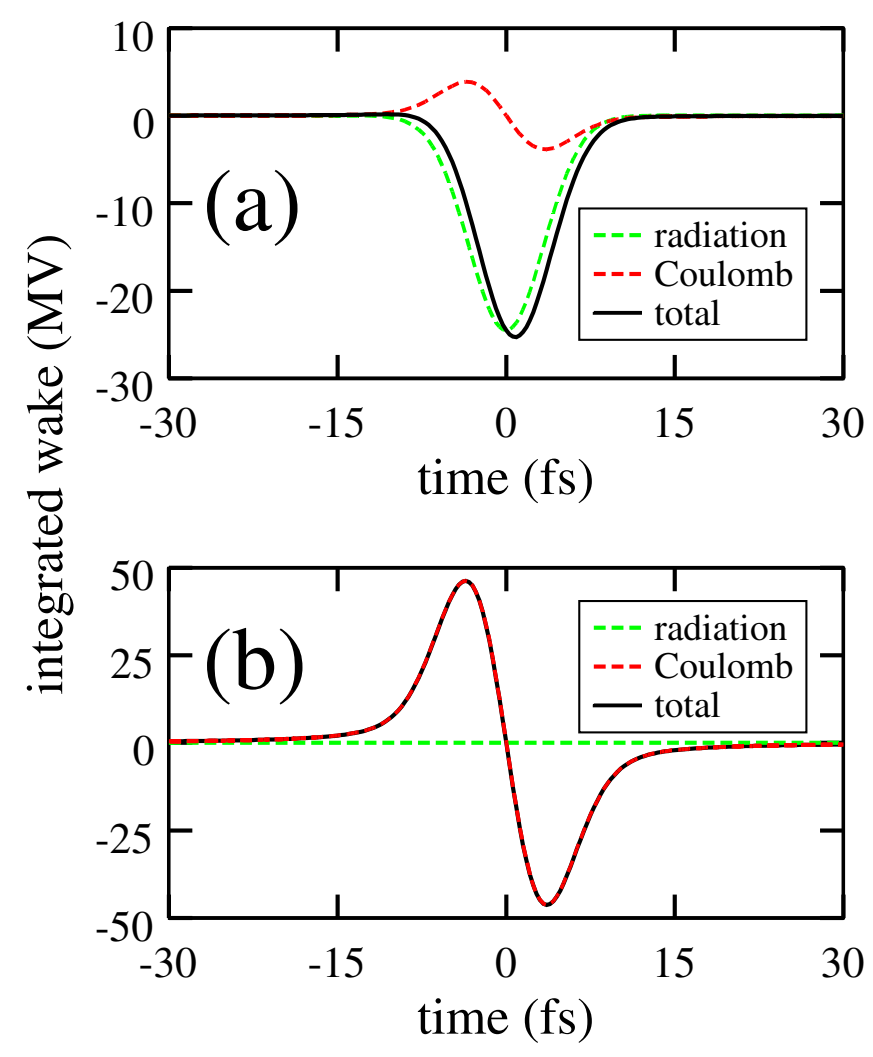

FIG. 3. (Color) The integrated coherent-radiation wake, Coulomb wake, and total wake for a bunch emitted from plasma with $r_{b}=\sigma_{z}=1 \mu \mathrm{m}$, peak current of $50 \mathrm{kA}$, and energy of $150 \mathrm{MeV}$. Here, a negative wake corresponds to energy loss; a positive time describes the tail of the bunch. (a) Integrated wakes at $z=3 \mathrm{~cm}$. (b) Integrated wakes through an undulator of length $0.8 \mathrm{~m}$, where the bunch radius is $r_{b}=30 \mu \mathrm{m}$.

Since the undulator is outside the radiation formation zone, the coherent-radiation wake within the undulator is approximately zero. The integrated wake through the undulator, approximated by the Coulomb wake for $r_{b}=$ $30 \mu \mathrm{m}$, is shown in Fig. 3(b).

Now, consider a tabletop x-ray FEL scenario in which a bunch with $r_{b} \approx \sigma_{z}=1 \mu \mathrm{m}$, peak current of $160 \mathrm{kA}$ and energy of $1.74 \mathrm{GeV}$ is emitted from plasma, and then focused over the region $4 \mathrm{~cm} \leq z \leq 14 \mathrm{~cm}$ to a collimated beam with $r_{b}=30 \mu \mathrm{m}$ and $\sigma_{z} \approx 1 \mu \mathrm{m}$ [12]. At $z=15 \mathrm{~cm}$, the bunch enters an undulator with length of $5 \mathrm{~m}$. The undulator is located within the radiation formation zone, whose length is $\sigma_{z} \gamma^{2}=11.6 \mathrm{~m}$. Figure 4(a) shows the integrated wakes at $z=3 \mathrm{~cm}, 1 \mathrm{~cm}$ upstream of the focusing triplet. The coherent-radiation wake exceeds that of space charge by a factor of $\sim 600$.

Since the undulator is inside the radiation formation zone, the coherent-radiation wake and the Coulomb wake both contribute to the integrated wake through the undulator, which is shown in Fig. 4(b). The coherent-radiation energy loss within the undulator may be prevented if the vacuum chamber shields the wake for $z>15 \mathrm{~cm}$, which

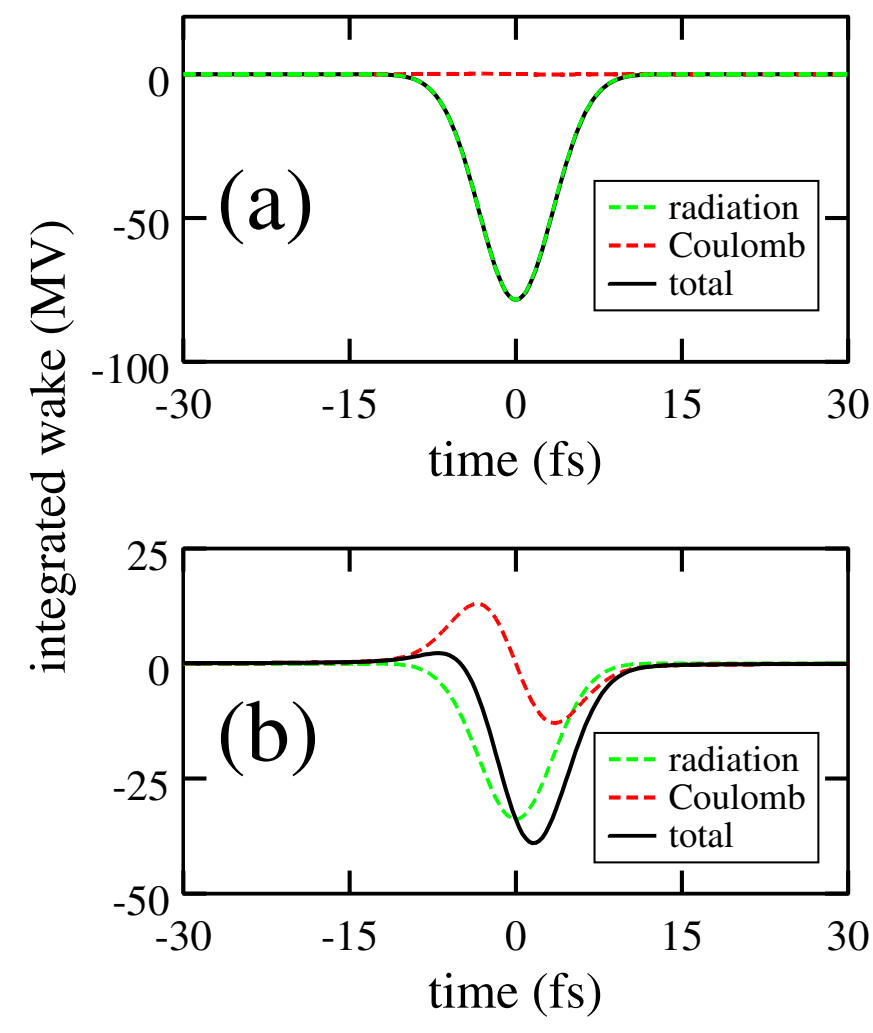

FIG. 4. (Color) The integrated coherent-radiation wake, Coulomb wake, and total wake for a bunch emitted from plasma with $r_{b}=\sigma_{z}=1 \mu \mathrm{m}$, peak current of $160 \mathrm{kA}$, and energy of $1.74 \mathrm{GeV}$. Here, a negative wake corresponds to energy loss; a positive time describes the tail of the bunch. (a) Integrated wakes at $z=3 \mathrm{~cm}$. (b) Integrated wakes through an undulator of length $5 \mathrm{~m}$, where the bunch radius is $r_{b}=30 \mu \mathrm{m}$.

requires a chamber height obeying $h^{2} / 2 \pi \sigma_{z}<15 \mathrm{~cm}$, i.e. $h<1 \mathrm{~mm}$. For a typical undulator gap of $1.7 \mathrm{~mm}$, shielding is expected for $z>46 \mathrm{~cm}$, reducing the integrated coherent-radiation wake through the undulator by $68 \%$. For a large undulator parameter, the wake from undulator radiation will also influence the bunch.

The wakes calculated above, which equal many MV, are sufficiently large that they may affect the FEL process [1].

\section{CONCLUSION}

We have calculated the longitudinal wake of a bunch of suddenly accelerated electrons in the radiation formation zone. This wake describes the wake of forward transition radiation, and it approximates the edge-radiation wake of a bunch exiting a bending magnet. We obtained a criterion that describes where this wake is larger than the Coulomb (longitudinal space charge) wake and developed expressions for the integrated wake. A comparison with the computed wake downstream of a bending magnet yields good agreement at distances exceeding the formation length of the bending magnet's ordinary synchrotron radiation. This suggests that our wake expressions may be 
used to approximate the wake of a bending magnet without numerical computation.

We considered the wake for schemes in which an ultrarelativistic bunch produced by laser-plasma acceleration exits the plasma and then drives a VUV or x-ray FEL. In these schemes, the integrated wakes from the bunch's coherent transition radiation and longitudinal space charge are many MV, sufficiently large to affect the FEL process.

\section{ACKNOWLEDGMENTS}

The author thanks G. Geloni for helpful correspondence, and J. J. Bisognano, M. A. Green, K. D. Jacobs, and K. J. Kleman for useful suggestions. This work is based upon research conducted at the Synchrotron Radiation Center, University of Wisconsin-Madison, which is supported by the National Science Foundation under Award No. DMR0537588.

[1] G. Geloni, E. Saldin, E. Schneidmiller, and M. Yurkov, DESY 06-222, 2006.

[2] R. A. Bosch and O. V. Chubar, in Synchrotron Radiation Instrumentation, edited by E. Fontes, AIP Conf. Proc. No. 417 (AIP, New York, 1997), p. 35.
[3] R. A. Bosch, Rev. Sci. Instrum. 73, 1423 (2002).

[4] J.D. Jackson, Classical Electrodynamics (Wiley, New York, 1975), 2nd ed., p. 688.

[5] Handbook of Mathematical Functions, edited by M. Abramowitz and I. A. Stegun (National Bureau of Standards, Washington, D.C., 1972), 10th printing, pp. 378-379, Eqs. 9.8.3 and 9.8.7.

[6] R. A. Bosch, Nucl. Instrum. Methods Phys. Res., Sect. A 482, 789 (2002).

[7] I.S. Gradshteyn and I. M. Ryzhik, Table of Integrals, Series, and Products (Academic Press, San Diego, 1994), 5th ed., p. 105, Eq. 2.271.3.

[8] E. L. Saldin, E. A. Schneidmiller, and M. V. Yurkov, Nucl. Instrum. Methods Phys. Res., Sect. A 398, 373 (1997), Eq. 75.

[9] G. Stupakov and P. Emma, in Proceedings of the Eighth European Particle Accelerator Conference, Paris (EPSIGA/CERN, Geneva, 2002), p. 1479.

[10] M. Dohlus and T. Limberg, Nucl. Instrum. Methods Phys. Res., Sect. A 393, 494 (1997).

[11] J. van Tilborg, C. B. Schroeder, C. V. Filip, Cs. Tóth, C. G. R. Geddes, G. Fubiani, E. Esarey, and W.P. Leemans, Phys. Plasmas 13, 056704 (2006).

[12] F. Grüner, S. Becker, U. Schramm, T. Eichner, M. Fuchs, R. Weingartner, D. Habs, J. Meyer-ter-Vehn, M. Geissler, M. Ferrario, L. Serafini, B. van der Geer, H. Backe, W. Lauth, and S. Reiche, Appl. Phys. B 86, 431 (2007). 